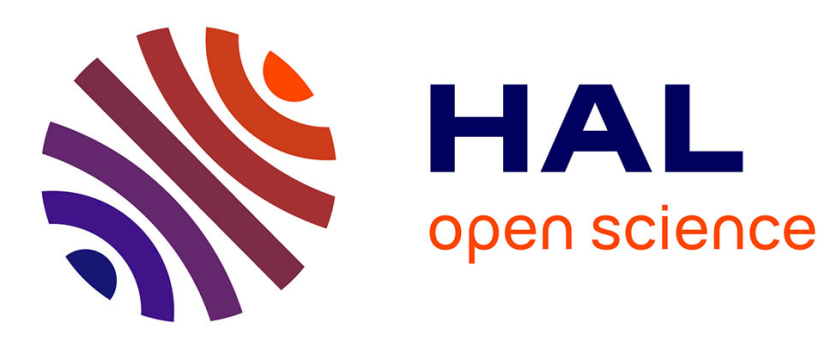

\title{
Selective Bond Cleavage in Informational Poly(Alkoxyamine Phosphodiester)s
}

Gianni Cavallo, Jean-louis Clément, Didier Gigmes, Laurence Charles, Jean-François Lutz

\section{To cite this version:}

Gianni Cavallo, Jean-louis Clément, Didier Gigmes, Laurence Charles, Jean-François Lutz. Selective Bond Cleavage in Informational Poly(Alkoxyamine Phosphodiester)s. Macromolecular Rapid Communications, 2020, 41 (12), pp.2000215. 10.1002/marc.202000215 . hal-02862144

\section{HAL Id: hal-02862144 \\ https://hal.science/hal-02862144}

Submitted on 30 Dec 2020

HAL is a multi-disciplinary open access archive for the deposit and dissemination of scientific research documents, whether they are published or not. The documents may come from teaching and research institutions in France or abroad, or from public or private research centers.
L'archive ouverte pluridisciplinaire HAL, est destinée au dépôt et à la diffusion de documents scientifiques de niveau recherche, publiés ou non, émanant des établissements d'enseignement et de recherche français ou étrangers, des laboratoires publics ou privés. 


\section{Selective bond cleavage in informational poly(alkoxyamine phosphodiester)s}

Gianni Cavallo, Jean-Louis Clément, Didier Gigmes, Laurence Charles,* Jean-François Lutz*

Published in Macromolecular Rapid Communications, 41, 2000215 (2020)

https://doi.org/10.1002/marc.202000215

Gianni Cavallo, Dr. Jean-François Lutz

Université de Strasbourg, CNRS, Institut Charles Sadron UPR22, 23 rue du Loess, 67034

Strasbourg Cedex 2, France.

E-mail: jflutz@unistra.fr

Prof. Dr. Jean-Louis Clément, Dr. Didier Gigmes, Prof. Dr. Laurence Charles

Aix Marseille Université, CNRS, UMR 7273, Institute of Radical Chemistry, 13397,

Marseille Cedex 20, France.

Email: laurence.charles@univ-amu.fr

\section{Abstract}

The collision-induced dissociation (CID) of sequence-defined poly(alkoxyamine phosphodiester)s was studied by electrospray ionization mass spectrometry. These informational polymers were synthesized using three different nitroxide building blocks, namely proxyl-, SG1- and TEMPO-derivatives. For a polymer containing TEMPO- and SG1based main chain alkoxyamines, it was found that both types of alkoxyamines break in CID tandem mass spectrometry (MS/MS). However, SG1-sites are preferentially cleaved and this predominance can be increased by reducing collision energy, even though selective bond fragmentation was not observed. On the other hand, for a polymer containing proxyl- and SG1alkoxyamines, selective bond cleavage was observed at all studied collision energies. The SG1alkoxyamines can be first cleaved in MS/MS conditions and secondly the proxyl-alkoxyamines in pseudo-MS ${ }^{3}$ conditions. These results open up interesting new avenues for the design of readable, erasable or programmable informational polymers. 


\section{Introduction}

Until very recently, macromolecular information storage was a property only exhibited by biopolymers. ${ }^{[1]}$ Yet, starting about seven years ago, ${ }^{[2]}$ synthetic information-containing macromolecules have been introduced as an exciting new class of functional polymers. ${ }^{[3-4]} \mathrm{Just}$ like biological polymers, these macromolecules store information in a defined comonomer sequence. ${ }^{[5-7]}$ Hence, this new field of research opens up unprecedented applications of synthetic polymers in areas such as data storage, ${ }^{[8-13]}$ cryptography, ${ }^{[14-15]}$ traceability, plastic recycling, ${ }^{[16]}$ anti-counterfeiting technologies, ${ }^{[17-18]}$ and synthetic Life. ${ }^{[19]}$ Overall, the synthesis of information-containing macromolecules is a topic that is increasingly studied and welldocumented. ${ }^{[4]}$ Comparatively, another fundamental aspect that has been less investigated is the degradation of these macromolecules; even though it is a crucial parameter that influences storage, ${ }^{[20]}$ erasure, ${ }^{[8]}$ editing ${ }^{[21]}$ and decryption of informational sequences. For instance, controlled bond cleavage is a crucial aspect in destructive sequencing methods such as collision-induced dissociation (CID) tandem mass spectrometry (MS/MS) or chemicallyinduced depolymerization. ${ }^{[22-25]}$ However, although all macromolecules are somehow decomposing over time, their degradation paths are not always controllable and useful. For example, the cleavage of DNA informational side-groups may occur in CID, thus prohibiting MS/MS sequencing. ${ }^{[26]}$ In this context, guiding macromolecular degradation through molecular design is an important objective. This aspect is, of course, much easier to implement in synthetic polymers than in biological ones because the molecular structure of the former can be varied at will, whereas the one of the latter is often limited by biological constraints.

Among the wide variety of chemical reactions and building blocks that can be used to construct synthetic information-containing polymers, the chemistry of nitroxides ${ }^{[27]}$ and alkoxyamines has been proven to be extremely useful. ${ }^{[8]}$ It is well known that NO-C bonds, formed by radical-radical coupling of nitroxides with carbon-centered radicals, are thermolabile and dynamic. Thus, various types of dynamic polymer architectures have been prepared 
using nitroxide chemistry. ${ }^{[28]}$ More recently, it was also shown that this chemistry can be used for the synthesis of digitally-encoded macromolecules such as poly(alkoxyamine amide)s ${ }^{[8,29]}$ and poly(alkoxyamine phosphodiester)s. ${ }^{[30-31]}$ Besides the fact that radical-radical coupling ${ }^{[32]}$ is a fast and efficient reaction for stepwise orthogonal polymer synthesis, ${ }^{[33]}$ the presence of alkoxyamine repeat units in the formed polymers enables chain erasing by thermal degradation $^{[8]}$ and favors sequencing because NO-C bonds are the first to break when subjected to CID. ${ }^{[30,34]}$ Thus, alkoxyamines were afterwards intentionally included in other types of digital polymers to guide their CID dissociation and sequencing. ${ }^{[35]}$ However, in all these works, the classic nitroxide 2,2,6,6-tetramethylpiperidinyloxy (TEMPO) was primarily used. Many other nitroxides exist and the stability of the corresponding alkoxyamines strongly depend on their molecular structure. ${ }^{[36-38]}$ For example, 2,2,5,5- tetramethylpiperidinyloxy (proxyl) and $N$ tert-butyl-1-diethylphosphono-2,2-dimethyl propyl (SG1) ${ }^{[39]}$ nitroxides lead to NO-C bonds, which are markedly different from TEMPO-based ones. ${ }^{[40]}$ In the present work, TEMPO, proxyl and SG1 building-blocks were tested for the iterative synthesis of digital poly(alkoxyamine phosphodiester)s and the MS/MS sequencing of the formed coded copolymers was investigated. In particular, the CID of the different types of alkoxyamines was examined and compared.

\section{Results and Discussion}

Scheme 1 shows the route that was used in this work for the synthesis of the sequence-defined poly(alkoxyamine phosphodiester)s. As described earlier, ${ }^{[30]}$ these polymers were prepared via an orthogonal iterative solid-phase approach involving two different types of bifunctional building blocks, namely a phosphoramidite monomer bearing an alkyl bromide and an hydroxyfunctional nitroxide, as well as two successive coupling reactions: (i) phosphoramidite coupling and (ii) radical-radical coupling. To include molecular information in the polymer chains, two different phosphoramidite monomers ( $\mathbf{0}$ and $\mathbf{1}$, Scheme 1) and three different nitroxides $(\mathbf{P}, \mathbf{S}$ 
and $\mathbf{T}$, Scheme 1) were used herein. In these syntheses, the radical-radical coupling step (ii) leads to the formation of main-chain alkoxyamines with the general structure $-\mathrm{NHCOC}\left(\mathrm{CH}_{3}\right)_{2}-$ ON-. The bond dissociation energy (BDE) of these alkoxyamines depends on the molecular structure of the corresponding nitroxide. For instance, it has been reported that for alkoxyamines formed with the same alkyl fragment $\mathrm{R}=\mathrm{C}_{6} \mathrm{H}_{5} \mathrm{C}\left(\mathrm{CH}_{3}\right)$, activation energy for the dissociation $E_{\text {ad }}$ at $293 \mathrm{~K}$ follows the trend R-SG1 < R-TEMPO < R-proxyl. ${ }^{[40]}$ Based on these literature data, building blocks $\mathbf{S}$ and $\mathbf{T}$ were first considered for the construction of sequencedefined poly(alkoxyamine phosphodiester)s. The model polymer P1 with sequence $\alpha$-0T0S0T was synthesized. Figure S1 shows the negative mode high-resolution electrospray ionization mass spectrometry (ESI-HRMS) spectrum recorded for this polymer. This measurement confirmed the synthesis of a uniform oligomer with a monoisotopic mass of $1574.8411 \mathrm{Da}$. The CID fragmentation of this polymer was then studied at different collision energies (Figure 1). As previously observed, ${ }^{[30]}$ alkoxyamines break preferentially in these structures, while other main-chain heteroatom-containing groups (i.e. amides and phosphates) remain stable. Yet, the fragmentation of the polymers strongly depends on collision energy (Figure 1a), with products of the SG1-based alkoxyamine (in blue) clearly formed from a lower energy threshold compared to those expected from $\mathrm{C}-\mathrm{ON}$ bond homolysis at the TEMPO moiety. As a result, using very low collision energies allows almost selective homolysis of the SG1-based alkoxyamine bond. For example, in the MS/MS spectrum recorded for $\mathbf{P 1}$ at $9 \mathrm{eV}$ (Figure 1b), the complementary products $\mathrm{c}_{2}{ }^{-2-}$ and $\mathrm{y}_{2}{ }^{--}$formed upon homolysis of the SG1-based alkoxyamine are observed with higher abundance than fragments $\mathrm{c}_{1}{ }^{\cdot 1-}$ and $\mathrm{y}_{3}{ }^{2-}$ generated after cleavage of the first TEMPO $\mathrm{C}-\mathrm{ON}$ bond (counting from left to right starting from the $\alpha$ terminus), while the $\mathrm{c}_{3}{ }^{-3-}$ ion expected at the second TEMPO-based alkoxyamine remains undetected. Raising collision energy up to $15 \mathrm{eV}$ allows both SG1- and TEMPO-based alkoxyamines to undergo homolytic cleavage. Consistent with the lack of transition state for bond homolysis in gas phase ions, the higher dissociation rate of the SG1-based alkoxyamine 
compared to TEMPO accounts for the larger abundance of the $\mathrm{c}_{2}{ }^{\cdot 2-} / \mathrm{y}_{2}{ }^{--}$ion pair compared to other fragments (Figure 1c). Nevertheless, these activation conditions allow all $\mathrm{c}_{\mathrm{i}}{ }^{\cdot{ }^{-}-}$fragments to form, hence enabling full sequence coverage by MS/MS according to decoding rules that have been previously established. ${ }^{[30]}$ Overall, a highly-favored (but not truly selective) decomposition of SG1-based alkoxyamines was observed as compared to TEMPO-based alkoxyamines in $\mathbf{P 1}$.

A second model polymer P2, having the sequence $\alpha$-1P1S0P1S, was then synthesized using the pair of building blocks $\mathbf{S}$ and $\mathbf{P}$. ESI-HRMS (Figure S2) confirmed the formation of the targeted polymer with a mass of 2119.1089 Da. Figure S3 shows the MS/MS breakdown curves recorded for P2. At all studied collision energies, the cleavage of SG1-based alkoxyamines is orthogonal as compared to proxyl-based ones. Of note, the experiment presented in Figure S3 was limited to collision energies below $15 \mathrm{eV}$ since activating $\mathbf{P 2}$ with higher energies induced secondary fragmentation via alternative rearrangement reactions. Figure 2a shows the MS/MS spectrum of $\mathbf{P 2}$ recorded at a collision energy of $9 \mathrm{eV}$, corresponding to a center-of-mass energy $\left(\mathrm{E}_{\mathrm{CM}}=0.470 \mathrm{eV}\right)$ comparable to that used for $\mathbf{P 1}$ in Figure $1 \mathrm{~b}\left(\mathrm{E}_{\mathrm{CM}}=0.473 \mathrm{eV}\right)$ for the sake of comparison. All observed peaks indicate that primary fragmentation of $\mathbf{P} 2$ proceeds at the two SG1-based alkoxyamines. Indeed, the peak at $\mathrm{m} / \mathrm{z} 309.1$ assigned to $\mathrm{c}_{1}{ }^{\cdot 1-}$ clearly corresponds to a secondary fragment formed from $\mathrm{c}_{2}{ }^{-2-}$ (vide infra) because the complementary $\mathrm{y}_{4}{ }^{3-}$ ion expected at $\mathrm{m} / \mathrm{z} 602.0$ in case of primary fragmentation of $\mathbf{P 2}$ is not detected. Data from Figure 2a show that other secondary dissociations selectively occur at SG1. This $\mathrm{C}-\mathrm{ON}$ bond cleavage in $\mathrm{c}_{4}{ }^{\cdot 4-}$ yields $\mathrm{c}_{2}{ }^{-2-}$ (hence further contributing the peak intensity at $m / z$ 399.7) together with the doubly charged internal fragment at $m / z 502.7$ (designated by a grey asterisk). The same $m / z 502.7$ internal fragment is also produced after the $\mathrm{y}_{3}{ }^{\cdot 3-}$ primary ion has eliminated the SG1-OH terminal radical moiety. Therefore, selective bond cleavage is more distinct for the pair SG1/proxyl than for SG1/TEMPO. These results are somehow in accordance with the homolysis rate constants 
reported for model alkoxyamines. ${ }^{[40]}$ Yet, it shall be reminded that the CID experimental conditions studied in this work are markedly different from solution conditions that are typically used in nitroxide mediated polymerization or in ESR decomposition studies. Furthermore, the homolytic cleavage of proxyl-, SG1- and TEMPO-based alkoxyamines is influenced by the nature of the alkyl fragment (i.e. the amide moiety - $\mathrm{NHCOC}\left(\mathrm{CH}_{3}\right)_{2}$ in the present case), by the steric and electronic effects of nitroxide substituents and eventually by side reactions. ${ }^{[36-37,41-}$ ${ }^{42]}$ Nonetheless, in the studied experimental conditions (that is, for gas phase ions in vacuum), SG1-based alkoxyamines were selectively cleaved in MS/MS regime where homolysis of C$\mathrm{ON}$ bonds at proxyl is not competitive. A second activation stage was required to induce cleavage of the proxyl-based alkoxyamines in primary fragments and hence recover the whole polymer sequence. To do so, $\mathrm{c}_{\mathrm{i}}{ }^{\mathrm{i}-}$ homolysis products of the two SG1 C-ON bonds were subjected to pseudo-MS ${ }^{3}$ experiments, after being formed in-source upon raising the kinetic energy of their transfer through the interface of the mass spectrometer. In such conditions, CID of $\mathrm{c}_{2}{ }^{\cdot 2-}$ at $m / z 399.2$ led to $\mathrm{c}_{1}{ }^{1-}$ at $m / z 309.1$ and $\mathrm{y}^{\prime \prime}{ }^{\cdot 1-}$ at $\mathrm{m} / \mathrm{z} 490.3$ (Figure 2b), indicating the $\alpha-1-\mathrm{P}-1$ starting block. Then, as illustrated in the inset dissociation scheme of Figure $2 \mathrm{c}$, the MS/MS pattern of $\mathrm{c}_{4}{ }^{4-}$ at $\mathrm{m} / \mathrm{z}, 451.2$ permitted to complete the sequence as $\alpha-1-\mathrm{P}-1-\mathrm{S}-0-\mathrm{P}-1$, and subtracting its $m / z$ value to the $m / z 528.8$ value of the $[\mathbf{P 2}-4 \mathrm{H}]^{4-}$ precursor confirmed the last building block was $\mathrm{S}-\mathrm{OH}$. Of note, selective homolysis of $\mathrm{C}-\mathrm{ON}$ bonds at $\mathrm{SG} 1$ is also observed in the pseudo-MS ${ }^{3}$ experiment reported in Figure 2c. Indeed, cleavage at the second proxyl group in $\mathrm{c}_{4}{ }^{4-}$ is not a primary dissociation event since the $\mathrm{c}_{3}{ }^{\cdot 3-}$ fragment expected at $\mathrm{m} / \mathrm{z}$ 438.2 is not detected. Instead, homolysis at this proxyl moiety occurs in $\mathrm{y}_{2} 2^{\cdot 2-}$ to produce $\mathrm{y}_{1}{ }^{\cdot 1-}$ (at the same $\mathrm{m} / \mathrm{z} 490.3$ compared to the internal fragment released from $\mathrm{c}_{2}{ }^{2-}$ to produce $\mathrm{c}_{1}{ }^{\cdot 1-}$ ) together with the $m / z 515.2$ internal fragment (annotated with an asterisk in Figure 2c). 


\section{Conclusions}

In summary, sequence-defined poly(alkoxyamine phosphodiester)s were synthesized using different nitroxide building blocks and their fragmentation was characterized by high-resolution electrospray ionization mass spectrometry. When the studied polymer contained both SG1- and TEMPO-based alkoxyamines, it was found that SG1 sites were predominantly (but not selectively) cleaved under CID conditions. Still, the preponderance of SG1-related fragments varied with collision energy. In case of a polymer containing both SG1- and proxyl-based alkoxyamines, selective bond cleavage was observed. First, the SG1-based sites were selectively cleaved in MS/MS and afterwards the proxyl alkoxyamines were cleaved in pseudo$\mathrm{MS}^{3}$ conditions. These findings open up interesting avenues for tuning the properties of digital polymers. For instance, they enable the design of informational macromolecules with programmed fragmentation; a strategy that has been recently validated for decoding long information sequences. $^{[35]}$ Alternatively, they may allow conception of blocky informational polymers, in which specific information blocks could be degraded and not others. Altogether, the results described in this communication further confirm that the properties of synthetic informational polymers can be finely tuned by macromolecular design.

\section{Acknowledgements}

These results were obtained with the support of the French National Research Agency (ANR project 00111001, grant numbers ANR-16-CE29-0004-01 and ANR-16-CE29-0004-02) and of the H2020 program of the European Union (project Euro-Sequences, H2020-MSCA-ITN-2014, grant agreement $\left.n^{\circ} 642083\right)$. The PhD position of G.C. was supported by the ITN EuroSequences. L.C. acknowledges support from Spectropole, the Analytical Facility of AixMarseille University, by allowing a special access to the instruments purchased with European Funding (FEDER OBJ2142-3341). 


\section{References}

[1] J.-F. Lutz, Macromolecules 2015, 48, 4759-4767.

[2] T. T. Trinh, L. Oswald, D. Chan-Seng, J.-F. Lutz, Macromol. Rapid Commun. 2014, 35, 141-145.

[3] H. Colquhoun, J.-F. Lutz, Nat. Chem. 2014, 6, 455-456.

[4] M. G. T. A. Rutten, F. W. Vaandrager, J. A. A. W. Elemans, R. J. M. Nolte, Nat. Rev. Chem. 2018, 2, 365-381.

[5] J.-F. Lutz, M. Ouchi, D. R. Liu, M. Sawamoto, Science 2013, 341, 1238149.

[6] J.-F. Lutz, Macromol. Rapid Commun. 2017, 38, 1700582.

[7] S. C. Solleder, R. V. Schneider, K. S. Wetzel, A. C. Boukis, M. A. R. Meier, Macromol. Rapid Commun. 2017, 38, 1600711.

[8] R. K. Roy, A. Meszynska, C. Laure, L. Charles, C. Verchin, J.-F. Lutz, Nat. Commun. 2015, 6, 7237.

[9] A. C. Boukis, M. A. R. Meier, Eur. Polym. J. 2018, 104, 32-38.

[10] S. Martens, A. Landuyt, P. Espeel, B. Devreese, P. Dawyndt, F. Du Prez, Nat. Commun. 2018, 9,4451 .

[11] Z. Huang, Q. Shi, J. Guo, F. Meng, Y. Zhang, Y. Lu, Z. Qian, X. Li, N. Zhou, Z. Zhang, X. Zhu, Nat. Commun. 2019, 10, 1918.

[12] J. M. Lee, M. B. Koo, S. W. Lee, H. Lee, J. Kwon, Y. H. Shim, S. Y. Kim, K. T. Kim, Nat. Commun. 2020, 11, 56.

[13] E. Laurent, J.-A. Amalian, M. Parmentier, L. Oswald, A. Al Ouahabi, F. Dufour, K. Launay, J.-L. Clément, D. Gigmes, M.-A. Delsuc, L. Charles, J.-F. Lutz, Macromolecules 2020, DOI: 10.1021/acs.macromol.1020c00666.

[14] A. C. Boukis, K. Reiter, M. Frölich, D. Hofheinz, M. A. R. Meier, Nat. Commun. 2018, 9, 1439.

[15] J. O. Holloway, F. Van Lijsebetten, N. Badi, H. A. Houck, F. E. Du Prez, Adv. Sci. 2020, $n / a, 1903698$.

[16] D. Karamessini, S. Poyer, L. Charles, J.-F. Lutz, Macromol. Rapid Commun. 2017, 38, 1700426.

[17] D. Karamessini, B. E. Petit, M. Bouquey, L. Charles, J.-F. Lutz, Adv. Funct. Mater. 2017, 27, 1604595.

[18] D. Karamessini, T. Simon-Yarza, S. Poyer, E. Konishcheva, L. Charles, D. Letourneur, J.-F. Lutz, Angew. Chem., Int. Ed. 2018, 57, 10574-10578.

[19] J.-F. Lutz, ACS Macro Lett. 2020, 9, 185-189. 
[20] R. N. Grass, R. Heckel, M. Puddu, D. Paunescu, W. J. Stark, Angew. Chem., Int. Ed. 2015, 54, 2552-2555.

[21] N. F. König, A. Al Ouahabi, L. Oswald, R. Szweda, L. Charles, J.-F. Lutz, Nat. Commun. 2019, 10, 3774.

[22] H. Mutlu, J.-F. Lutz, Angew. Chem., Int. Ed. 2014, 53, 13010-13019.

[23] P. Edman, Acta Chem. Scand. 1950, 4, 283-293.

[24] B. Liu, Q. Shi, L. Hu, Z. Huang, X. Zhu, Z. Zhang, Polym. Chem. 2020, 11, 1702-1707.

[25] S. D. Dahlhauser, P. R. Escamilla, A. N. VandeWalle, J. T. York, R. M. Rapagnani, J. S. Shei, S. A. Glass, J. N. Coronado, S. R. Moor, D. P. Saunders, E. V. Anslyn, J. Am. Chem. Soc. 2020, 142, 2744-2749.

[26] C. M. Castleberry, L. P. Rodicio, P. A. Limbach, Curr. Protoc. Nucleic Acid Chem. 2008, 35, 10.12.11-10.12.19.

[27] L. Tebben, A. Studer, Angew. Chem., Int. Ed. 2011, 50, 5034-5068.

[28] T. Maeda, H. Otsuka, A. Takahara, Prog. Polym. Sci. 2009, 34, 581-604.

[29] R. K. Roy, C. Laure, D. Fischer-Krauser, L. Charles, J.-F. Lutz, Chem. Commun. 2015, $51,15677-15680$.

[30] G. Cavallo, A. Al Ouahabi, L. Oswald, L. Charles, J.-F. Lutz, J. Am. Chem. Soc. 2016, $138,9417-9420$.

[31] G. Cavallo, S. Poyer, J.-A. Amalian, F. Dufour, A. Burel, C. Carapito, L. Charles, J.-F. Lutz, Angew. Chem., Int. Ed. 2018, 57, 6266-6269.

[32] J. Kulis, C. A. Bell, A. S. Micallef, Z. Jia, M. J. Monteiro, Macromolecules 2009, 42, 8218-8227.

[33] T. T. Trinh, C. Laure, J.-F. Lutz, Macromol. Chem. Phys. 2015, 216, 1498-1506.

[34] L. Charles, C. Laure, J.-F. Lutz, R. K. Roy, Macromolecules 2015, 48, 4319-4328.

[35] A. Al Ouahabi, J.-A. Amalian, L. Charles, J.-F. Lutz, Nat. Commun. 2017, 8, 967.

[36] S. Marque, C. Le Mercier, P. Tordo, H. Fischer, Macromolecules 2000, 33, 4403-4410.

[37] S. Marque, H. Fischer, E. Baier, A. Studer, J. Org. Chem. 2001, 66, 1146-1156.

[38] D. Bertin, D. Gigmes, S. R. A. Marque, P. Tordo, Macromolecules 2005, 38, 26382650 .

[39] S. Grimaldi, J.-P. Finet, F. Le Moigne, A. Zeghdaoui, P. Tordo, D. Benoit, M. Fontanille, Y. Gnanou, Macromolecules 2000, 33, 1141-1147.

[40] S. Marque, J. Org. Chem. 2003, 68, 7582-7590. 
[41] N. R. Cameron, C. A. Bacon, A. J. Reid, in Advances in Controlled/Living Radical Polymerization, Vol. 854, American Chemical Society, 2003, pp. 452-465.

[42] G. Gryn'ova, C. Y. Lin, M. L. Coote, Polym. Chem. 2013, 4, 3744-3754. 


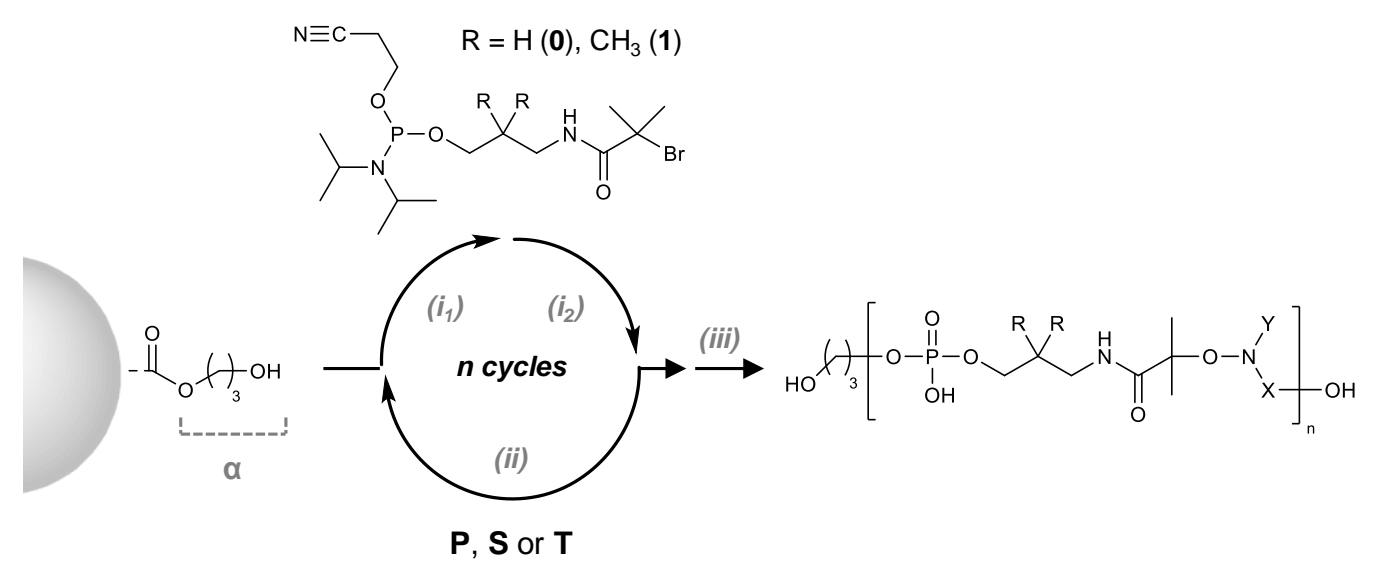

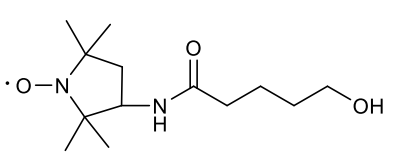

(P)

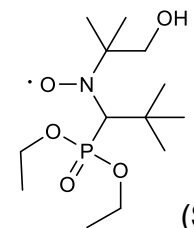

$(\mathbf{S})$

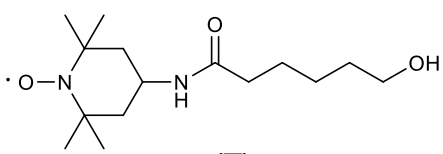

( $\mathbf{T})$

Scheme 1. Iterative steps and building blocks used in the present work for the synthesis of sequence-defined poly(alkoxyamine phosphodiester)s. Experimental conditions: $\left(i_{1}\right)$ phosphoramidite coupling: RT, AcCN, tetrazole; $\left(i_{2}\right)$ oxidation: $\mathrm{RT}, \mathrm{I}_{2}, 2$,6-lutidine, $\mathrm{THF} / \mathrm{H}_{2} \mathrm{O}$; (ii) radical-radical coupling: $\mathrm{CuBr}, \mathrm{Me}_{6} \mathrm{TREN}, \mathrm{DMSO}$, (iii) cleavage: piperidine, AcCN, RT, then $\mathrm{MeNH}_{2}, \mathrm{NH}_{4} \mathrm{OH}, \mathrm{H}_{2} \mathrm{O}$, RT. 


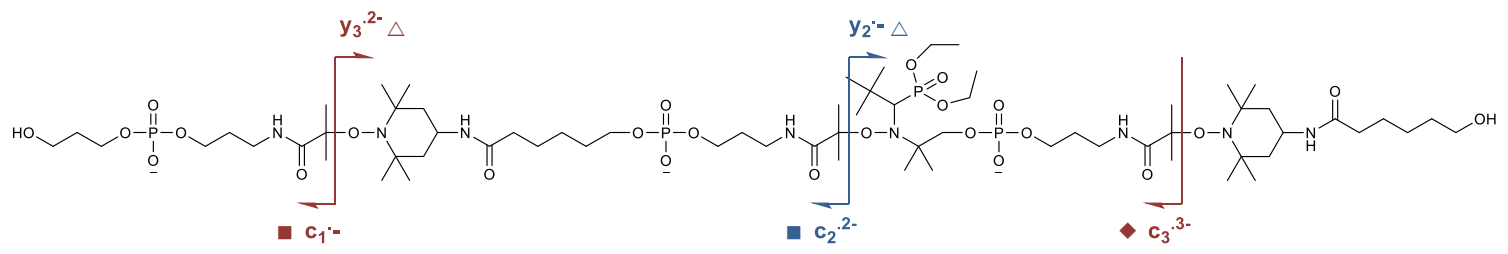

a.

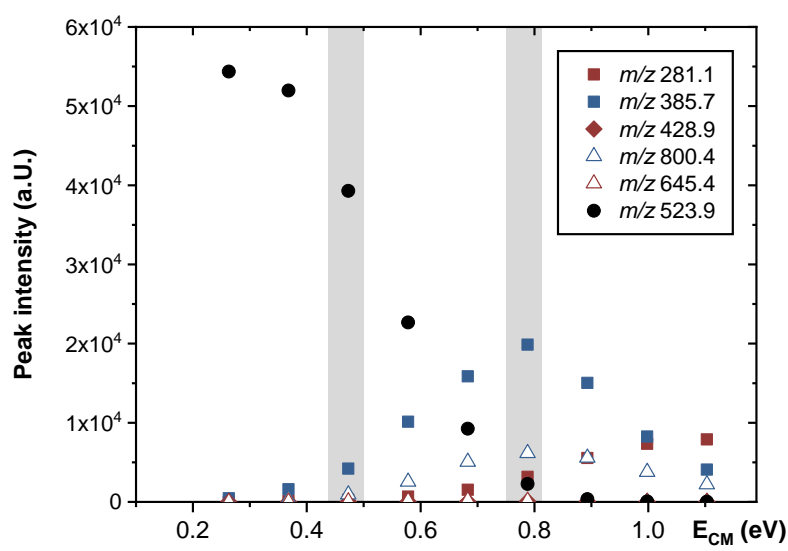

b.

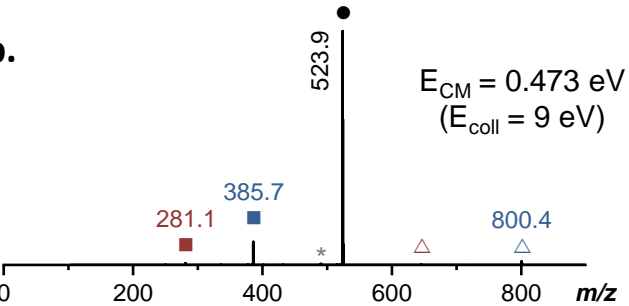

c.

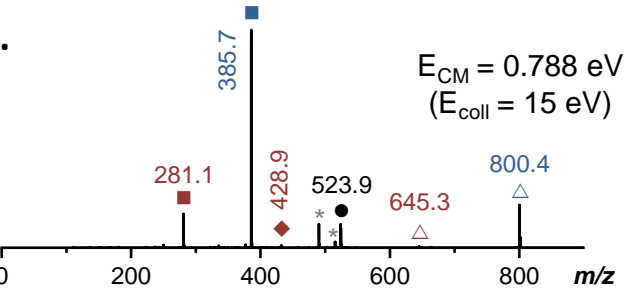

Figure 1. MS/MS fragmentation of P1. (a) Breakdown curves recorded for the $[\mathbf{P 1}-3 \mathrm{H}]^{3-}$ precursor ion at $m / z 523.9$ (black dots) at different collision energies. The grey zones highlight the examples that are displayed in panels $\mathbf{b}$ and c. (b) MS/MS spectrum recorded for $[\mathbf{P 1}-3 \mathrm{H}]^{3-}$ at a laboratory frame collision energy of $9 \mathrm{eV}(0.473 \mathrm{eV}$ in the center-of-mass frame). (c) MS/MS spectrum recorded for $[\mathbf{P 1}-3 \mathrm{H}]^{3-}$ at a laboratory frame collision energy of $15 \mathrm{eV}(0.788$ $\mathrm{eV}$ in the center-of-mass frame). The grey asterisks designate internal fragments. 

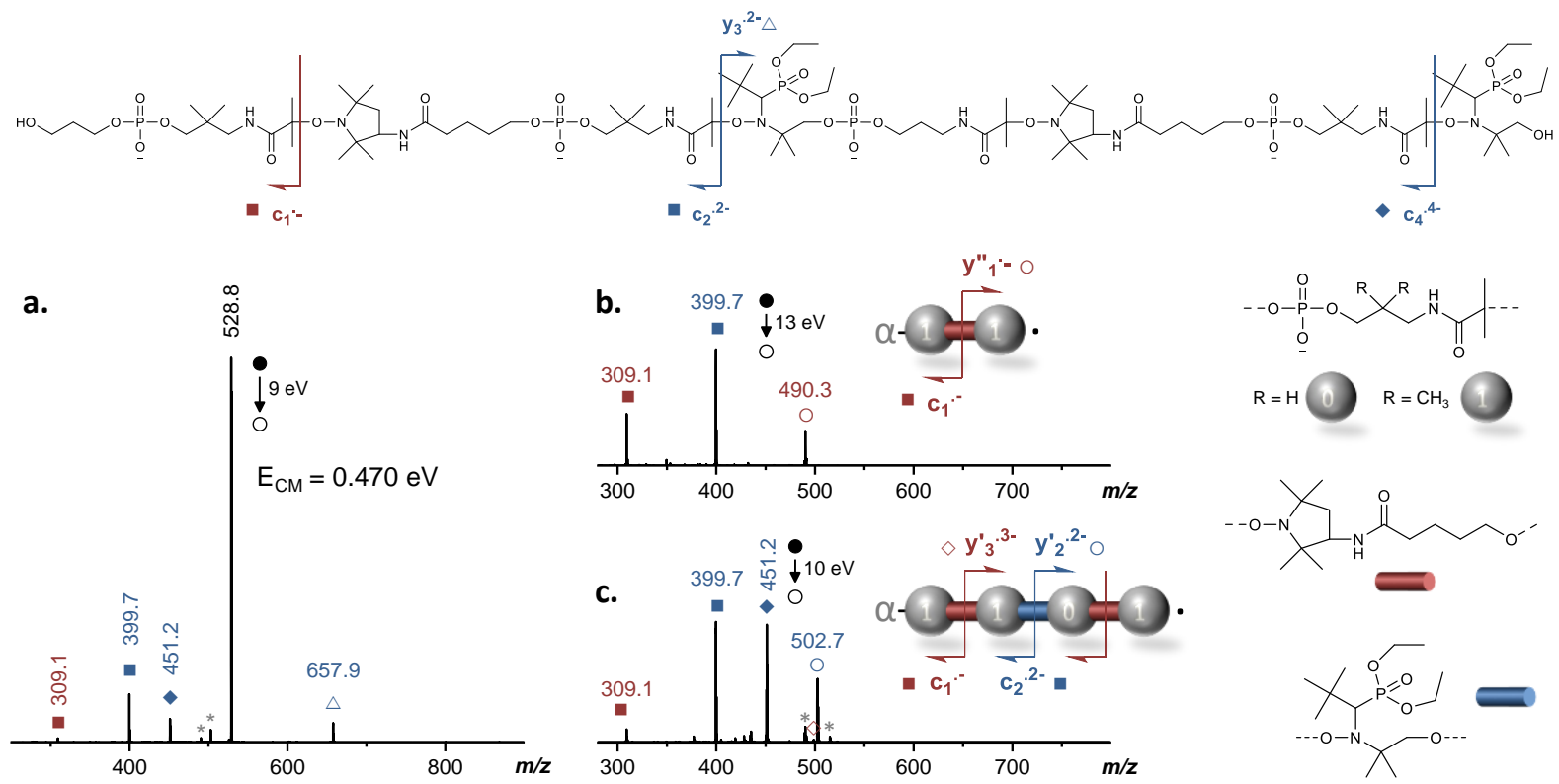

Figure 2. Successive MS/MS and pseudo-MS ${ }^{3}$ fragmentation of P2. (a) MS/MS spectrum recorded for $[\mathbf{P 2}-4 \mathrm{H}]^{4-}$ at a laboratory frame collision energy of $9 \mathrm{eV}(0.478 \mathrm{eV}$ in the centerof-mass frame). The grey asterisks designate internal fragments. (b) Pseudo-MS ${ }^{3}$ spectrum recorded for the $\mathrm{c}_{2}{ }^{-2-}$ precursor ion at a $13 \mathrm{eV}$ collision energy (laboratory frame). (c) Pseudo$\mathrm{MS}^{3}$ spectrum recorded for the $\mathrm{c}_{4}{ }^{\cdot 4-}$ precursor ion at a $10 \mathrm{eV}$ collision energy (laboratory frame). The grey asterisks designate internal fragments. 


\section{Supporting Information}

\section{Selective bond cleavage in informational poly(alkoxyamine phosphodiester)s}

Gianni Cavallo, Jean-Louis Clément, Didier Gigmes, Laurence Charles, Jean-François Lutz*

Materials. 3-Amino-proxyl (99\%, Acros Organics), N,N-diisopropylethylamine (DIPEA, 99\%, TCI), 1H-benzotriazol-1-yloxytripyrrolidinophosphonium hexafluorophosphate (PyBOP, 98\%, Novabiochem/Merck), copper(I) bromide (98\%, Alfa Aesar), tris(2-dimethylaminoethyl)amine (Me ${ }_{6}$ TREN, 99\%, Alfa Aesar), piperidine (99\%, Alfa Aesar), methylamine (aqueous solution, $40 \%$, Fluka), ammonium hydroxide solution $\left(28-30 \% \mathrm{NH}_{3}, \mathrm{VWR}\right)$, tetrazole (acetonitrile solution, $0.45 \mathrm{M}$, Sigma-Aldrich), tetrabutylammonium fluoride solution (1.0 M in THF, Sigma-Aldrich), 2,6-lutidine (98\%, Alfa Aesar), tetrahydrofuran (THF, > 99\%, 250 ppm BHT, Sigma-Aldrich), anhydrous dichloromethane (DCM, Sigma-Aldrich), anhydrous acetonitrile (AcCN, Sigma-Aldrich) and anhydrous dimethyl sulfoxide (DMSO >99\%, Sigma-Aldrich) were used as received. Anhydrous tetrahydrofuran (THF) was obtained on a dry solvent station GTS100. 5-((triisopropylsilyl)oxy)pentanoic acid was synthesized following a previously described procedure. ${ }^{[1]}$ The nitroxides $\mathbf{S}, \mathbf{T}$ and the phosphoramidite monomers $\mathbf{0}, \mathbf{1}$ were prepared as previously reported. ${ }^{[2-3]}$ The phosphoramidite monomers were kept in the freezer at $-18^{\circ} \mathrm{C}$. Oligomers iterative syntheses were performed in an equipped argon/vacuum solid phase extraction glass tube (12 mL with frit 3) and stirred with an IKA HS 260 Basic shaker. They were performed on an aminomethylated-polystyrene resin $\left(1.4 \mathrm{mmol} \mathrm{g}^{-1}\right.$, Merck) that was modified with 4-oxo-4-(3-(dimethoxy-trityloxy)propoxy)butanoic acid as reported earlier. ${ }^{[1]}$

Synthesis of 5-hydroxy-N-proxyl-pentanamide. The nitroxide $\mathbf{P}$ was synthesized by reacting 3-amino-proxyl with 5-((triisopropylsilyl)oxy)pentanoic acid and subsequent deprotection of the triisopropylsilyl (TIPS) group. 5-((Triisopropylsilyl)oxy)pentanoic acid (190 mg, 0.66 mmol, 1 eq.), DIPEA (255mg, 1.98 mmol, 3 eq.) and 3-amino-proxyl (103 mg, 0.66 mmol, 1 eq.) were dissolved in $20 \mathrm{~mL}$ of anhydrous $\mathrm{DCM}$ and the solution was cooled to $0^{\circ} \mathrm{C}$. To the reaction mixture, PyBOP (515 mg, $0.99 \mathrm{mmol}, 1.5$ eq.) was added and the solution was allowed to reach room temperature and stirred overnight. The day after, the reaction was quenched with ammonium chloride saturated solution and the solvent was evaporated. The aqueous layer was extracted with EtOAc $(3 \times 20 \mathrm{~mL})$, washed with brine $(20 \mathrm{~mL})$ and dried over MgSO4. Chromatographic column on silica gel (EtOAc/Cyclohexane) (30/70) gives the TIPS-protected product in 67\% yield. ESIHRMS $[\mathrm{M}+\mathrm{H}]^{+}, m / z$ expected for $\mathrm{C}_{23} \mathrm{H}_{46} \mathrm{~N}_{2} \mathrm{O}_{3} \mathrm{Si}^{\cdot+}$ 414.3272, found 414.3271. The TIPSprotected intermediate $(353 \mathrm{mg}, 0.85 \mathrm{mmol}, 1$ eq.) was then dissolved in10 $\mathrm{mL}$ of THF. To the solution, $3.0 \mathrm{~mL}$ of TBAF (1M in THF, $3.0 \mathrm{mmol}, 3.5$ eq.) was added. The reaction was monitored 
by thin layer chromatography and was stopped after 2.5 hours. The solvent was evaporated and purified by chromatographic column on silica gel (dichloromethane/methanol) (95/5). After purification, the $\mathrm{OH}$-functional nitroxide $\mathbf{P}$ was recovered in $98 \%$ yield as a red yellow powder. ESI-HRMS $[\mathrm{M}+\mathrm{H}]^{+}, m / z$ expected for $\mathrm{C}_{13} \mathrm{H}_{26} \mathrm{~N}_{2} \mathrm{O}_{3}{ }^{*+} 258.1938$, found 258.1936.

Solid-phase iterative synthesis of the oligo(alkoxyamine phosphodiester)s. The protocol for the solid-phase iterative synthesis of oligo(alkoxyamine phosphodiester)s was reported in a previous publication. ${ }^{[1]}$ In brief, the phosphoramidite coupling (step $\left(i_{1}\right)$ in Scheme 1) was performed using 3 eq. of monomer $\mathbf{0}$ or $\mathbf{1}$ for 30 minutes on $120 \mathrm{mg}$ of modified resin (estimated loading of $0.7 \mathrm{mmol} \mathrm{g} \mathrm{g}^{-1}$ after modification). This reaction is always followed by an oxidation step using a $0.1 \mathrm{M}$ iodine solution (step $\left(i_{2}\right)$ in Scheme 1). The radical-radical coupling step (step (ii) in Scheme 1) was performed using 5 eq. of nitroxide $\mathbf{S}, \mathbf{T}$ or $\mathbf{P}$ for 40 minutes. Iterative cycles $\left(i_{1}\right),\left(i_{2}\right)$ and $(i i)$ were repeated a certain number of times until a desired chain-length and primary structure were reached. After synthesis, the oligomers were deprotected and cleaved using first a piperidine solution in acetonitrile followed by a treatment with a mixture of ammonia aqueous solution, methylamine aqueous solution and 1,4 dioxane (step (iii) in Scheme 1). Yield: $85 \%$ (P1), $70 \%$ (P2).

Electrospray Ionization Mass Spectrometry. High resolution MS, MS/MS and pseudo-MS ${ }^{3}$ experiments were performed using a QqTOF mass spectrometer (QStar Elite, SCIEX, Concord, ON, Canada) with the ESI source operated in the negative mode (capillary voltage: $-4200 \mathrm{~V}$; cone voltage: $-75 \mathrm{~V}$ for MS and MS/MS, $-200 \mathrm{~V}$ for pseudo-MS ${ }^{3}$ ). Ions were accurately mass measured in the orthogonal acceleration time-of-flight (oa-TOF) mass analyzer, using the precursor ions as internal standards to calibrate MS/MS data. In this instrument, air was used as nebulizing gas (10 psi) while nitrogen was used as curtain gas (20 psi) and collision gas. Instrument control, data acquisition and data processing were achieved using Analyst software (QS 2.0) provided by Applied Biosystems. Polymer samples (a few mg) were first solubilized in methanol (SDS, Peypin, France) and then diluted (1/10 to 1/1000, v/v) in methanol supplemented with ammonium acetate (Sigma Aldrich, Saint Louis, MO) at a $3 \mathrm{mM}$ concentration level, prior to injection in the ESI source at $10 \mu \mathrm{L} / \mathrm{min}$ using a syringe pump. 


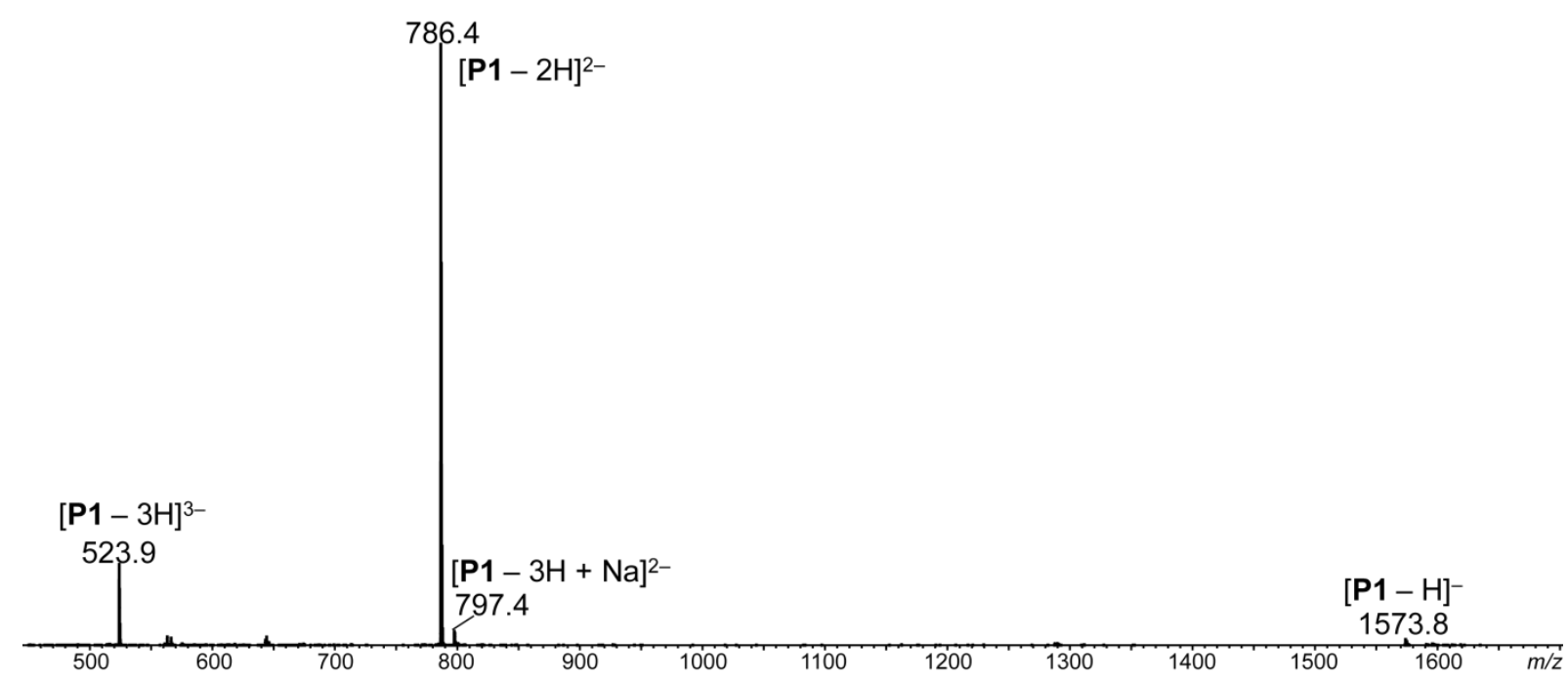

Figure S1. Negative mode ESI-HRMS recorded for polymer P1 with sequence $\alpha$-0T0S0T. P1 was accurately mass measured in the positive ion mode as doubly protonated molecule at $\mathrm{m} / \mathrm{z}$ 788.4276, that is, with a $-0.3 \mathrm{ppm}$ error compared to the $\mathrm{m} / z .788 .4278$ value calculated for $\mathrm{C}_{67} \mathrm{H}_{136} \mathrm{~N}_{8} \mathrm{O}_{25} \mathrm{P}_{4}{ }^{2+}$.

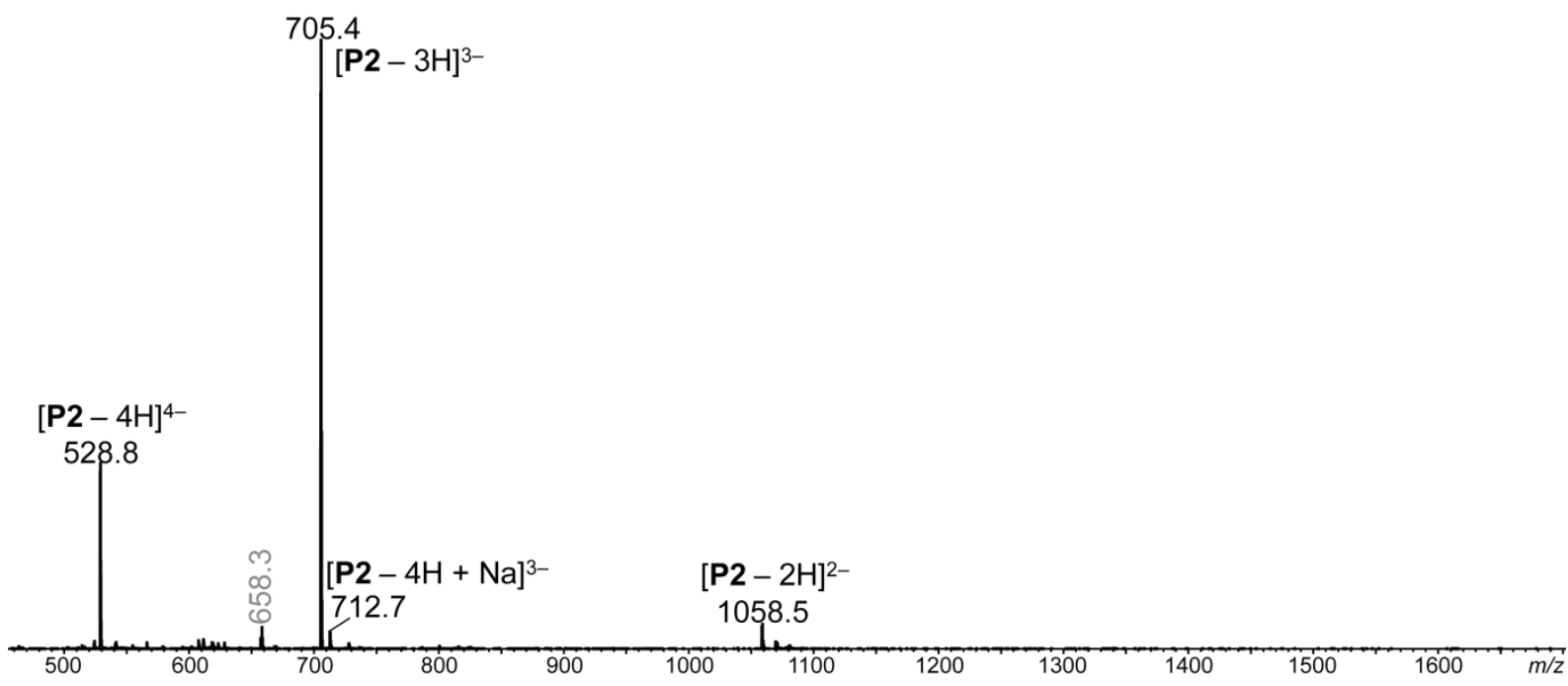

Figure S2. Negative mode ESI-HRMS recorded for polymer P2 with sequence $\alpha-1 \mathrm{P} 1 \mathrm{~S} 0 \mathrm{P} 1 \mathrm{~S}$. $\mathbf{P 2}$ was accurately mass measured in the positive ion mode as doubly protonated molecule at $m / z 1060.5618$, that is, with a $+0.2 \mathrm{ppm}$ error compared to the $m / z 1060.5617$ value calculated for $\mathrm{C}_{89} \mathrm{H}_{182} \mathrm{~N}_{10} \mathrm{O}_{34} \mathrm{P}_{6}{ }^{2+}$. 

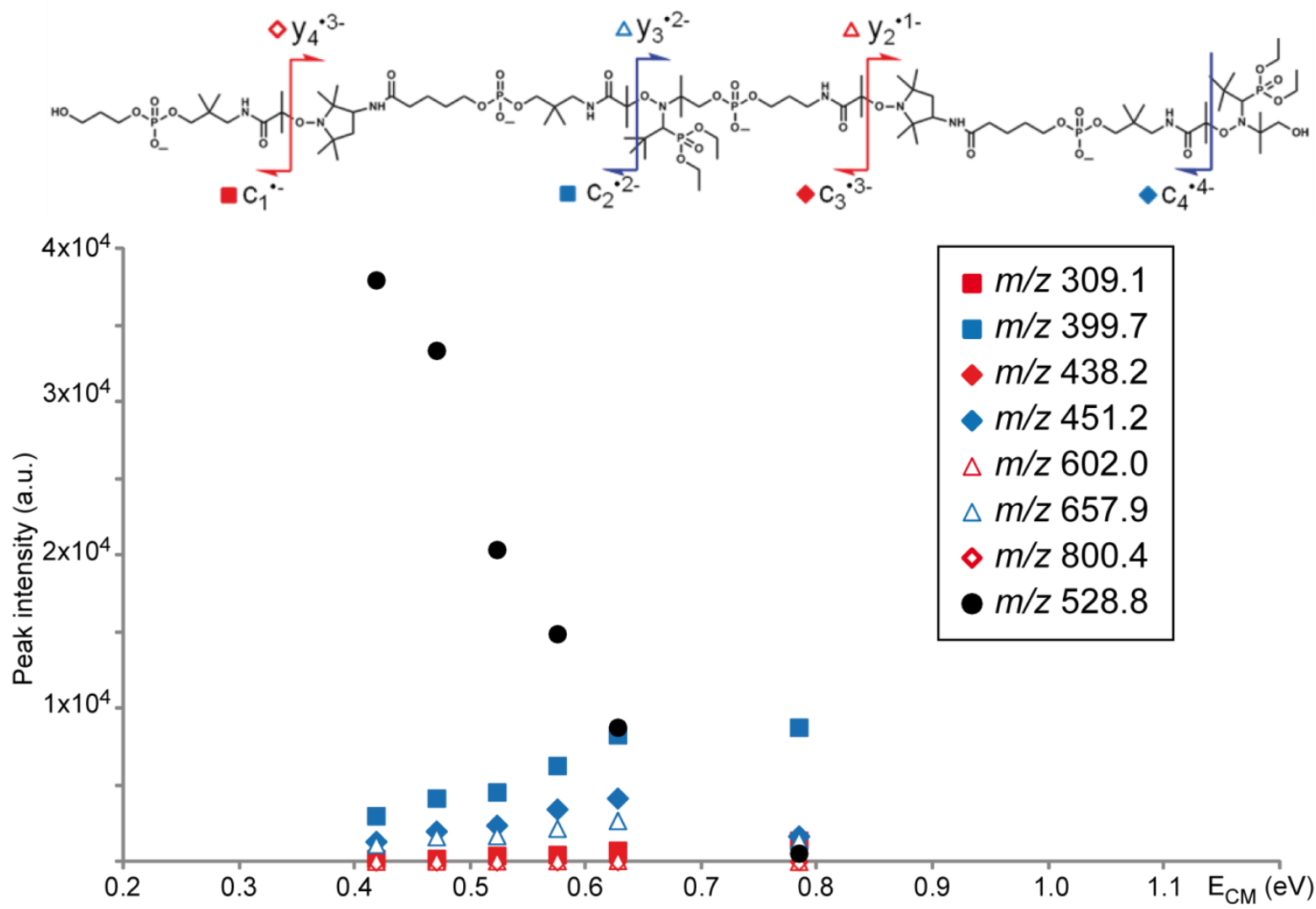

Figure S3. Breakdown curves obtained for $[\mathbf{P 2}-4 \mathrm{H}]^{4-}$ at $\mathrm{m} / z 528.8$ (black dots). 


\section{References}

[1] G. Cavallo, S. Poyer, J.-A. Amalian, F. Dufour, A. Burel, C. Carapito, L. Charles, J.-F. Lutz, Angew. Chem., Int. Ed. 2018, 57, 6266-6269

[2] S. Acerbis, D. Bertin, B. Boutevin, D. Gigmes, P. Lacroix-Desmazes, C. Le Mercier, J.F. Lutz, S. R. A. Marque, D. Siri, P. Tordo, Helv. Chim. Act. 2006, 89, 2119-2132

[3] G. Cavallo, A. Al Ouahabi, L. Oswald, L. Charles, J.-F. Lutz, J. Am. Chem. Soc. 2016, $138,9417-9420$ 